\title{
EFFECT OF ALPHA IRRADIATION ON SILICON SCHOTTKY DIODE DETECTOR
}

\author{
Niharika. R. Sukhrani ${ }^{1}$, Achintya Das ${ }^{2}$, Prakash Vaidya ${ }^{3}$, Siddhartha. P. Duttagupta ${ }^{4}$ \\ ${ }^{1}$ Student of M.E. (Instrumentation \& Control Engg.), VESIT, Chembur, Mumbai, India \\ ${ }^{2}$ PhD Student, Electrical Engineering Department, Indian Institute of Technology Bombay, Powai, India \\ ${ }^{3}$ Head of Department, Instrumentation Department, VESIT, Chembur, India \\ ${ }^{4}$ Assistant Professor, Electrical Engineering Department, Indian Institute of Technology Bombay, Powai, India
}

\begin{abstract}
This paper gives a brief overview of Semiconductor Schottky diode detector in response to $\alpha$-particle irradiation. As $\alpha$-particle are difficult to detect so we need a high resolution device for its detection. In this paper we have simulated a model of Si Schottky diode detector in Sentaurus TCAD Software. TCAD (Technology Computer Aided Design) simulation is a boon for the semiconductor devices. Using this tool we can simulate and design semiconductor devices and can generate simulation model to get the best optimum simulation results for semiconductor diode. Sentaurus TCAD supports wide range of semiconductor technologies from conventional to compound semiconductor detector. $\alpha$-particles induced transient current pulse measurement were carried out on Si Schottky diode at (1) different temperature, (2) different energies of the incident $\alpha$-particle and (3) different reverse bias of Schottky diode. Effect of temperature, incident $\alpha$-particle and reverse bias on the generated $\alpha$-induced transient current pulse were analyzed.
\end{abstract}

Keywords: Semiconductor Schottky diode, Single event transient (SET), $\alpha$ particle, reverse bias, Sentaurus TCAD.

$* * *$

\section{INTRODUCTION}

Semiconductor detectors have been part of the Nuclear Spectroscopy for the past several years. They have a great advantage in the $\mathrm{x}$-ray and gamma ray spectroscopy applications. Semiconductor detectors have been widely used in nuclear physics applications in the various forms such as pixel detectors, detector pads etc. This report, describes the behavior of the Silicon detectors which are exclusively used for charged particle detection. Semiconductor detectors are also referred as solid state detectors. Earlier these detectors were called as crystal counters. These detectors offers several advantages over other kinds of detectors that is scintillator and gas-filled detectors such as faster response time and smaller size [1].The average energy required to produce a single electron-hole pair is $3.6 \mathrm{eV}$ for Silicon [2], which is less as that compared to scintillator detector and gas filled detectors. As higher number of electron-hole pairs are generated in semiconductor detector this leads to better energy resolution [3].

Fabrication of the defect and trap free crystalline Silicon substrate is economical [4]. Thus these high purity crystalline substrate have little interference with the signal carriers, hence such silicon detectors are excellent for charged particle detection [5].

This paper mainly focuses on detection of $\alpha$ particles, as they are extremely harmful to environment as well as to human beings. If $\alpha$ emitters such as radon and its progeny are inhaled or ingested, then the emitted $\alpha$ particles can create biological damage of tissues and cells. They can induce dreadful diseases to human beings such as cancer [6].
Due to high linear energy transfer (LET) of $\alpha$ particle, a thin sheet of paper or a thin layer of dust (few hundredths of millimeter) is sufficient to stop $\alpha$ particle [7]. So thus detection of $\alpha$ particle is of primary importance and it is difficult to detect. The application of $\alpha$ particle detectors include uses in smoke detectors, radon detection.

$\alpha$ particle spectroscopy can be utilized to detect and quantify the presence of different kinds of $\alpha$ emitters [8]. High resolution devices are required for $\alpha$ particle detection.

In this paper we have developed a model of Silicon Schottky Diode in Sentaurus TCAD software to detect $\alpha$ particles $[8,9]$. Senaturus TCAD is one such software provided by Synopsys. When the $\alpha$ particle interacts with the active area of the Schottky diode detector it creates an electron hole pair cloud and subsequently the reverse bias of the diode separates the electron hole pairs and generate electronic pulse to detect the $\alpha$ particle. Here in this report, $\alpha$ induced current pulses were analysed at 1) Different temperature, 2) Different reverse bias and 3) Different energies of $\alpha$ particles.

\section{DETECTOR DESCRIPTION}

Fig 1 illustrates the cross section of the Si Schottky diode detector. The Detector consists of p-type Si layer with boron doping concentration of $5 \times 10^{15} \mathrm{~cm}^{-3}$. As shown in fig 1 , a $\mathrm{SiO}_{2}$ layer was realized on top of the $\mathrm{Si}$ substrate with a 30 um wide opening for Schottky contact. Here $\mathrm{SiO}_{2}$ layer was used as guard rings. The main advantage of using guard ring is to reduce the leakage current and in improving the breakdown voltage of the Si detectors [10]. Ohmic contact is realized on its bottom surface. 


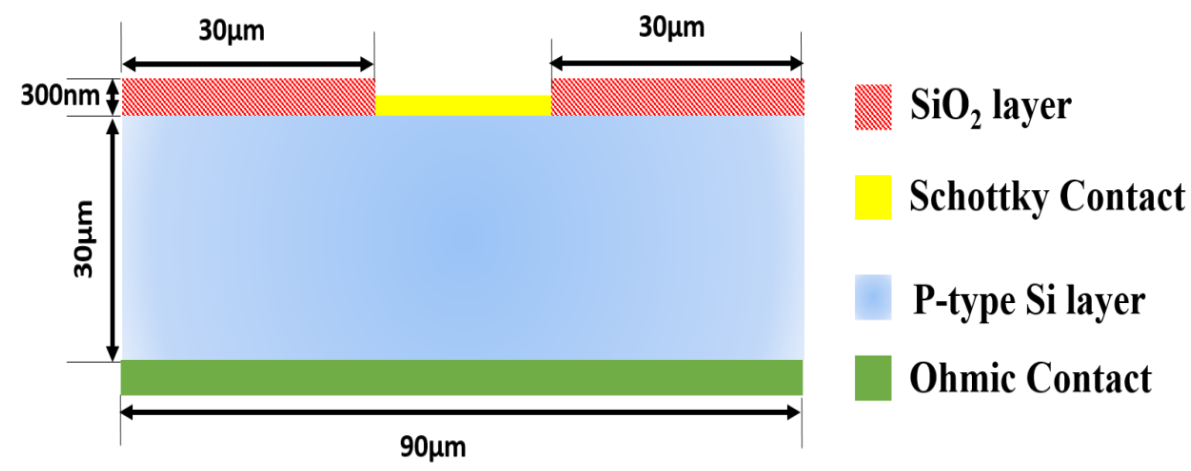

Fig. 1. Schematic diagram of Si based $\alpha$ detector

\section{PHYSICAL MODEL}

The key models that are used for Silicon Schottky diode in the simulation are discussed in this section. The important physical models that were used in this simulation are listed as below 1) Hydrodynamic Model, 2) Bandgap, 3) Mobility, 4) Recombination, and 5) Alpha particle $(\alpha)$ radiation Model.

\subsection{Hydrodynamic Model}

The model that describes the transport of electrons and phonons is the classical hydrodynamic model. Hydrodynamic models play an important role in simulating electron transport in semiconductor devices [11].

\subsection{Bandgap Model}

The Bandgap narrowing is an important phenomenon in semiconductor physics and the equation of Temperature dependent bandgap is as follows (1).

$$
E_{g}=E_{g}(0)-\frac{\alpha T 2}{T+\beta}
$$

Where $\operatorname{Eg}(0)$ is the bandgap energy at $0 \mathrm{~K}$.

Table 1: Simulation Parameter For Bandgap Model [I3]

\begin{tabular}{|l|l|l|}
\hline Parameter & Value & Unit \\
\hline$\alpha$ & $4.73 * 10^{-4}$ & $\mathrm{eV} / \mathrm{K}$ \\
\hline$\beta$ & 636 & $\mathrm{~K}$ \\
\hline $\mathrm{E}_{\mathrm{g}}(0)$ & 1.1679 & $\mathrm{eV}$ \\
\hline
\end{tabular}

\subsection{Mobility Model}

Mobility and carrier concentration are temperature dependent. The factors behind the reduction of mobility is as follows: 1) High Electric Field 2) High Temperature 3) Higher Doping Levels.

At higher electric field, as electrons gain higher energy than that of the applied electric field, they emit optical phonons thus mobility decreases, as the scattering increases [12].

At higher temperature, the mobility decreases due to increase in scattering and phonon generation. [12].
For doped material that is as impurities are introduced in the semiconductor the carrier scatters with the impurities that leads to degradation of mobility [12].

The default model considered by Sentaurus Device for doping-dependent mobility is Masetti model for Silicon detector [12] [13] as given by (2). For Silicon Detector, the equation for the High field dependent mobility model is given below in (4).

$$
\begin{aligned}
\mu_{\text {dop }}=\mu_{\min 1} \exp & \left(-\frac{P_{C}}{N_{A_{,},}+N_{D_{,},}}\right)+\frac{\mu_{\text {const }}-\mu_{\min 2}}{1+\left(\frac{N_{A_{,} 0}+N_{D_{1} 0}}{C_{r}}\right)^{\alpha}} \\
& -\frac{\mu_{1}}{1+\left(\frac{C_{S}}{N_{A_{,},}+N_{D_{,},}}\right)^{\beta}}
\end{aligned}
$$

where

$$
\begin{aligned}
& \mu_{\text {const }}=\mu_{\max }\left(\frac{T}{T_{0}}\right)^{-\mu \exp } \\
& \mu_{\text {high }}=\frac{\mu_{\text {low }}}{\left(1+\left(\frac{\mu_{\text {low }} E}{V_{\text {sat }}}\right)^{\beta 1}\right)^{\beta 1}}
\end{aligned}
$$

where

$$
\begin{aligned}
& \beta 1=\beta_{0}\left(\frac{T}{T_{0}}\right)^{\beta \exp } \\
& V_{\text {sat }}=V_{\text {sat0 }}\left(\frac{T}{T_{0}}\right)^{\text {Vsatexp }}
\end{aligned}
$$

Table 2: Simulation Parameter For Mobility Model [13]

\begin{tabular}{|l|l|l|l|}
\hline Parameter & Electron & Hole & Unit \\
\hline$\mu_{\min 1}$ & 52.2 & 44.9 & $\mathrm{~cm}^{2} / \mathrm{V}-\mathrm{s}$ \\
\hline$\mu_{\min 2}$ & 52.2 & 0.0 & $\mathrm{~cm}^{2} / \mathrm{V}-\mathrm{s}$ \\
\hline$\mu_{1}$ & 43.4 & 29.0 & $\mathrm{~cm}^{2} / \mathrm{V}-\mathrm{s}$ \\
\hline $\mathrm{P}_{\mathrm{c}}$ & 0 & $9.23 * 10^{16}$ & $\mathrm{~cm}^{-3}$ \\
\hline $\mathrm{C}_{\mathrm{r}}$ & $9.68 * 10^{16}$ & $2.23 * 10^{17}$ & $\mathrm{~cm}^{-3}$ \\
\hline $\mathrm{C}_{\mathrm{s}}$ & $3.43 * 10^{20}$ & $6.10^{*} 10^{20}$ & $\mathrm{~cm}^{-3}$ \\
\hline$\alpha$ & 0.680 & 0.719 & 1 \\
\hline$\beta$ & 2.0 & 2.0 & 1 \\
\hline
\end{tabular}




\subsection{Recombination Model}

Carrier Lifetime is an important parameter in semiconductor physics [16]. The carrier lifetime decreases nearly linearly as doping increases [15]. In silicon, as recombination lifetime is function of dopant concentration it can be very well described by Schokley-Read-Hall (SRH recombination model).

Schokley-Read-Hall (SRH) recombination rate is given by the following equation (7)

$$
R_{S R H}=\frac{n p-\left(n_{\text {i eff }}\right)^{2}}{\tau_{p}\left(n+n_{1}\right)+\tau_{n}\left(p+p_{n}\right)}
$$

where,

$$
\begin{aligned}
& n 1=n_{i, \text { eff }}\left(\frac{E_{\text {trap }}}{k T}\right) \\
& p 1=n_{i, \text { eff }}\left(-\frac{E_{\text {trap }}}{k T}\right)
\end{aligned}
$$

Here Etrap indicates the difference between the defect level and intrinsic level. The value of Etrap $=0$ for silicon detector.

Doping dependence and temperature dependence of SRH recombination life time modeled stated in the Sentaurus Device with the Scharfetter relation is given as below by (9) and (10) respectively.

$$
\begin{gathered}
\tau_{\text {dop }}=\tau_{\text {min }}+\frac{\tau_{\text {max }}-\tau_{\text {min }}}{1+\left(\frac{N_{A 0}+N_{D} 0}{{ }^{\prime} \text { Nref }}\right)^{\gamma}} \\
\tau(T)=\tau_{0}\left(\frac{T}{T_{0}}\right)^{\alpha}
\end{gathered}
$$

Auger Recombination is the process in which recombination of hole and electron takes place but instead of emitting a photon or phonon the energy is transferred to the electron and hole [15]. For band to band transition Auger Recombination is considered in our report. The factor that limits the carrier lifetime is the Auger Recombination Process [17].

Auger recombination is modeled by the following equation (11)

$$
R_{A}=C_{n}(T) n+C_{p}(T) p
$$

where

$$
\begin{gathered}
c_{n}(T)=\left(A_{A, n}+B_{A, n}\left(\frac{T}{T_{0}}\right)+C_{A, n}\left(\frac{T}{T_{0}}\right)^{2}\right)[1 \\
+H_{n}(e)^{\left.\frac{-n}{N o n}\right]}
\end{gathered}
$$

$$
\begin{gathered}
c_{p}(T)=\left(A_{A, p}+B_{A, p}\left(\frac{T}{T_{0}}\right)+C_{A, p}\left(\frac{T}{T_{0}}\right)^{2}\right)[1 \\
\left.+H_{p}(e)^{\frac{-p}{N o p}}\right]
\end{gathered}
$$

Table 3:Simulation Parameter for recombination Model[13]

\begin{tabular}{|l|l|l|l|}
\hline Parameter & Electron & Hole & Unit \\
\hline$\tau_{\min }$ & 0 & 0 & $\mathrm{sec}$ \\
\hline$\tau_{\max }$ & $1 * 10^{-5}$ & $3 * 10^{-6}$ & $\mathrm{sec}$ \\
\hline $\mathrm{N}_{\mathrm{ref}}$ & $5 * 10^{16}$ & $5 * 10^{16}$ & $\mathrm{~cm}^{-3}$ \\
\hline$\gamma$ & 1 & 1 & 1 \\
\hline Etrap & 0 & $\mathrm{eV}$ \\
\hline$\alpha$ & 1.72 & 1.72 & \\
\hline $\mathrm{A}_{\mathrm{An}, \mathrm{p}}$ & $6.7 * 10^{-32}$ & $7.2^{*} 10^{-32}$ & $\mathrm{~cm}^{6} / \mathrm{s}$ \\
\hline $\mathrm{B}_{\mathrm{An}, \mathrm{p}}$ & $2.45^{*} 10^{-31}$ & $4.5^{*} 10^{-33}$ & $\mathrm{~cm}^{6} / \mathrm{s}$ \\
\hline $\mathrm{C}_{\mathrm{An}, \mathrm{p}}$ & $-2.2^{*} 10^{-32}$ & $2.63^{*} 10^{-32}$ & $\mathrm{~cm}^{6} / \mathrm{s}$ \\
\hline
\end{tabular}

\subsection{Alpha Particle ( $\alpha$ ) Radiation Model}

When alpha particles $(\alpha)$ are incident on Silicon Schottky diode then electron-hole pairs are generated in detector along the particle track [18]. The generation rate of electronhole pairs due to alpha particle $(\alpha)$ of energy E can be written by (14).

If $\mathrm{u}<\alpha_{1}+\alpha_{2}$

$$
\begin{aligned}
& G(u, v, w, t) \\
& =\frac{a}{s \cdot \sqrt{2 \pi}} \exp \left[-\frac{1}{2}\left(\frac{t-t_{m}}{s}\right)^{2}-\frac{1}{2}\left(\frac{v^{2}-w^{2}}{w_{t}^{2}}\right)^{2}\right]\left[C_{1} e^{\alpha u}\right. \\
& \left.+c_{2} \exp \left(-\frac{1}{2}\left(\frac{u-\alpha_{1}}{\alpha_{2}}\right)^{2}\right)\right]
\end{aligned}
$$

Else if $\mathrm{u} \geq \alpha_{1}+\alpha_{2}$

$$
G(u, v, w, t)=0
$$

The maximum Bragg peak $\alpha_{l}$ is stated by the equation (15)

$$
\alpha_{1}=a_{0}+a_{1} E+a_{2} E^{2}
$$

Table 4: Simulation Parameter For Alpha Radiation Model[14]

\begin{tabular}{|l|l|l|}
\hline Parameter & Value & Unit \\
\hline $\mathrm{s}$ & $2 * 10^{-12}$ & $\mathrm{Sec}$ \\
\hline $\mathrm{w}_{\mathrm{t}}$ & $1 * 10^{-5}$ & $\mathrm{~cm}$ \\
\hline $\mathrm{c}_{2}$ & 1.40 & 1 \\
\hline$\alpha$ & 90.0 & $\mathrm{~cm}^{-1}$ \\
\hline$\alpha_{2}$ & $5.5^{*} 10^{-4}$ & $\mathrm{~cm}$ \\
\hline$\alpha_{3}$ & $2.0^{*} 10^{-4}$ & $\mathrm{~cm}$ \\
\hline $\mathrm{E}_{\mathrm{p}}$ & 3.6 & $\mathrm{eV}$ \\
\hline $\mathrm{a}_{0}$ & $-1.033^{*} 10^{-4}$ & $\mathrm{~cm}$ \\
\hline $\mathrm{a}_{1}$ & $2.7 * 10^{-10}$ & $\mathrm{~cm} / \mathrm{eV}^{-17}$ \\
\hline $\mathrm{a}_{2}$ & $4.33^{*} 10^{-17}$ & $\mathrm{~cm} / \mathrm{eV}^{2}$ \\
\hline
\end{tabular}




\section{ALPHA IRRADIATION}

Si Schottky Diode detector is irradiated with $\alpha$ particles of different energies of $1 \mathrm{MeV}, 2 \mathrm{MeV}, 3 \mathrm{MeV}, 4 \mathrm{MeV}, 5 \mathrm{MeV}$, $6 \mathrm{MeV}, 7 \mathrm{MeV}$ for testing the detector at different conditions such as at different temperature and different reverse bias.

When $\alpha$ particles impinges on the Silicon region of the detector, we collected a sequence of $\alpha$ induced transient current pulses at reverse bias Si Schottky diode detector.

\section{RESULTS AND DISCUSSIONS}

The $\alpha$ induced current pulse was observed on the Si detector. The measurement were carried out at temperature ranging from $300^{\circ} \mathrm{K}$ to $350^{\circ} \mathrm{K}$.

\subsection{Response of Detector with different $\alpha$ Particle}

\section{Energy}

We analyze the response of different energies of $\alpha$ particles by keeping the other parameters constant that is temperature and reverse bias are kept constant.

In the Fig 2 shown below, the pulse height increases for the $\alpha$ particle ranging from $1 \mathrm{MeV}$ to $6 \mathrm{MeV}$, but the pulse height decreases for $7 \mathrm{MeV} \alpha$ particle. The reason behind that pulse height won't increase infinitely as the energy increases is stated as below. When the energy increases electron-hole pair density increases but external reverse bias induced field won't be able to separate the high density electron hole pair cloud.

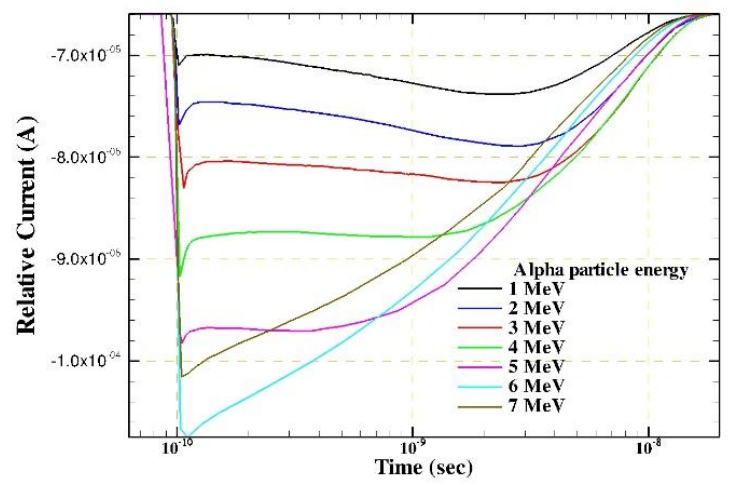

Fig. 2. Plot of Transient current pulse with different energy of the incident $\alpha$ particles.

\subsection{Reverse Bias}

When the reverse bias increases the charge collection time will decrease but the pulse height will increase.

Higher the bias voltage, the width of the active region increases thus resulting in larger pulse heights. The total charge liberated by the $\alpha$ particles is produced entirely in the active region of the detector and can be measured. When the reverse bias increase the leakage current will also increase. Fig 3, illustrates when the reverse (back) bias is varied from $-1 \mathrm{~V}$ to $-4 \mathrm{~V}$, we observe that the width is reduced.

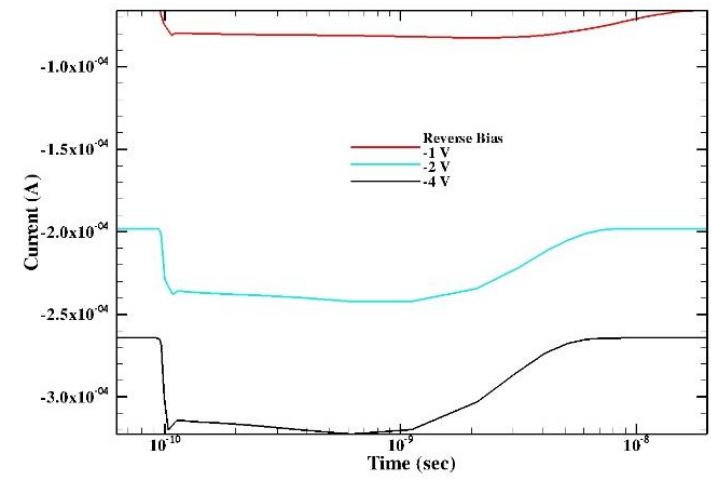

Fig. 3. Plot of Transient current pulse with different reverse bias condition

\subsection{Effect of Temperature}

When the temperature increases the charge collection time will increase but the pulse height will decrease, as the phonon generation will increase and the rate of recombination will also increase and the mobility of electron hole pair will decrease.

Fig 4 depicts that the carrier velocity decreases once the temperature increases from room temperature $300^{\circ} \mathrm{K}$ to $350^{\circ} \mathrm{K}$. The reason behind this that atoms have more thermal vibrations which increase the rate of phonon generation. Due to the carrier scattering with the generated phonon, mobility will decrease and subsequently the pulse height will decrease and pulse width will increase [17] .
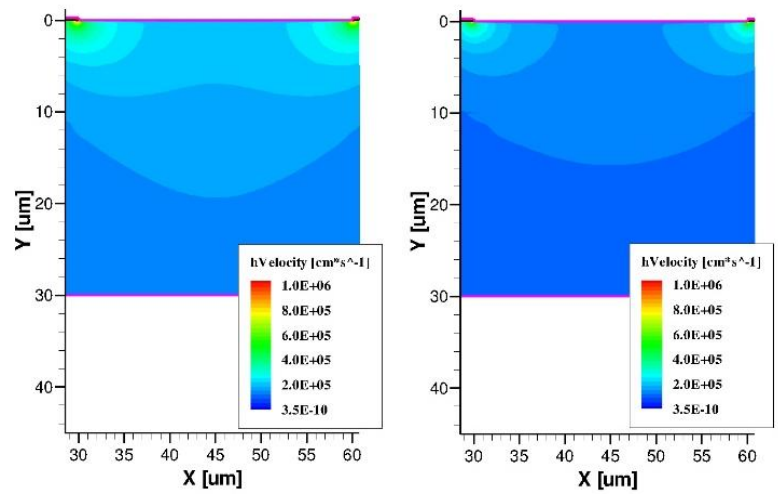

Fig. 4. Plot of Carrier Concentration with respect to temperature

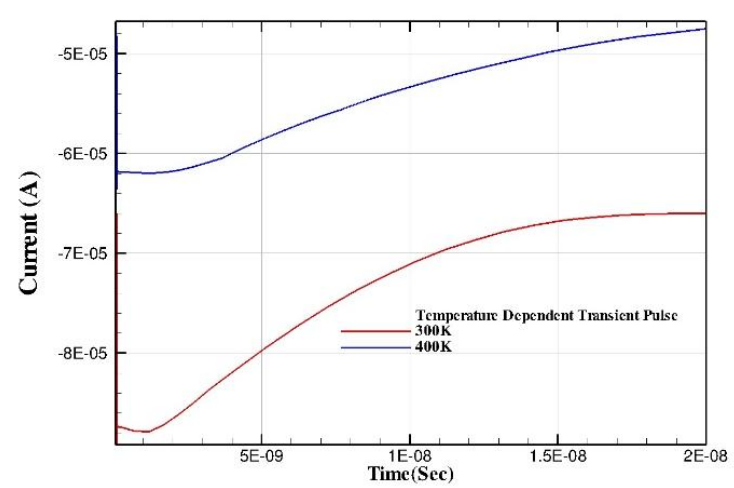

Fig. 5. Plot of Transient current pulse at different temperature 


\section{CONCLUSION}

We have thus studied and observed the effect of temperature, reverse bias on Silicon Semiconductor diode detector. The lattice scattering phenomenon is dominant in semiconductors for higher temperatures [18]. We have thus successfully developed a silicon semiconductor model in TCAD software and proved our concept that the detector is capable of detecting $\alpha$ particles even at higher temperature of $350^{\circ} \mathrm{K}$.

\section{ACKNOWLEDGEMENTS}

We would like to acknowledge IITBNF facility for providing the Sentaurus Software in the CEN MCL lab.

\section{REFERENCES}

[1] G.F.Knoll, Radiation Detection and Measurement, John Wiley \&Sons, USA, 1979.

[2] G.D. Watkins and J.W.Corbett, "Defects in Irradiated Silicon: Electron Paramagnetic Resonance and Electron-

[3] Nuclear Double Resonance of the Si-E Center", Physical Review Vol. 134, Number 5A, 1 JUNE 1964.

[4] T. Takahashi, S. Watanabe, "Recent progress in CdTe and CdZnTe detectors", Nuclear Science, IEEE Transactions on, Vol.48, no.4, pp.950-959, 2001.

[5] W.C. O'Mara, R. B Haber and L.P. Hunt, "Handbook of Semiconductor Silicon Technology" Noyes Publication, 1990.

[6] D. K. Wehe, "Current Trends In Ionizing Radiation Detection", Nuclear Engineering and Technology, Vol.38 No.4 June 2006.

[7] M. Kreuzer, B.Grosche , M.Schnelzer, A.Tschense , F. Dufey , L.Walsh, "Radon and risk of death from cancer and cardiovascular diseases in the German uranium miners cohort study: follow-up" 1946-2003, Radiation and Environmental Biophysics, Vol.45, No. 2, pp. 177-185, 2010.

[8] http/www.acq.osd.mil/ncbdp/narp/Radiation_Data/R adiation_Detection_and_Measurement.html

[9] Ortec, AN34 Experiment 4 "Alpha Spectroscopy with

surfacebarrierdetectors"http:www3.nd.edu/ wzech/A pplication-Note-AN\#\$-Experiments-Nuclear-

Science-Experiments-4.pdf

[10] A. Das, S.P.Duttagupta, M.N. Gandhi, "TCAD, Compact Models for SiC Schottky Diode as Neutron Induced Ion Detector, International Conference on Electronics, Communication and Signal Processing", 2012, Nagpur.

[11] V. Mishra,V D Srivastava and S K Kataria, "Role of guard rings in improving the performance of Silicon Detectors" , Pramana Indian Academy of Sciences Vol. 65, No. 2, August 2005, physics pp.259-272.

[12] T Grasser, T W Tang, H Kosina, and S Selberherr, "A Review of Hydrodynamic and Energy Transport Models for Semiconductor Device Simulation",
Proceedings of the IEEE, Vol.91, No.2, February 2003.

[13] S.N Mohammad, A. V.Bemis , R.L.Carter, and R.B.Renbeck, "Temperature, electric field, and doping dependent mobilities of electrons and holes in semiconductors", Solid-State Electronics, Volume 36, Issue 12, December 1993, pp. 1677-1683

[14] Sentaurus Device User Manual, Synopsys Version F2011.09, September 2011.

[15] G.Masetti, M.Severi , and S.Solmi , "Modeling of Carrier Mobility Against Carrier Concentration in Arsenic-, Phosphorus-, and Boron-Doped Silicon", IEEE Transactions on Electron Devices, Vol. ED-30, no. 7, pp. 764-769, 1983.

[16] J.P.BValenzuela, Development of innovative Silicon radiations Detectors.

[17] E. Gaubasa and J. Vanhellemontb , "Comparative Study of Carrier Lifetime Dependence on Dopant Concentration in Silicon and Germanium", Journal of The Electrochemical Society, Vol. 154, issue 3 ppH231 H238 2007.

[18] Soo Han Kim Han , Hwan Se Park and Jang Ho Ha "Characteristics of Silicon Surface Barrier Radiation Detectors for $\alpha$ particle detection", Journal of the Korean Physical Society, Vol. 52, No. 6, June 2008, pp. 1754-1758.

[19] G.P.Neudeck., R.S.Okojie, and L.Y.Chen, "High Temperature Electronics A Role for Wide Bandgap

[20] Semiconductors?" Proceedings of the IEEE, Vol.90, No. 6, June 2002. 